\title{
How Do We Deal with Uncertain Information? Effects of Verbal and Visual Expressions of Uncertainty on Learning
}

\author{
Manuela Glaser $^{1}$ (D) $\cdot$ Dominik Lengyel $^{2} \cdot$ Catherine Toulouse $^{2} \cdot$ Stephan Schwan $^{1}$
}

Accepted: 17 January 2022 / Published online: 31 January 2022

(c) The Author(s) 2022

\begin{abstract}
Based on the knowledge generation model for visual analytics including uncertainty propagation and human trust building (Sacha et al. 2016), the cognitive theory of multimedia learning (Mayer, 2014), the multimedia principle (Butcher, 2014), and previous studies on the effects of different uncertainty visualization styles, an integrated theoretical approach is proposed to examine the influence of different degrees of information uncertainty and different uncertainty visualization styles on processing pictures of two archeological reconstructions with accompanying audio explanations presented in a multimedia learning environment. A $4 \times 3$ design with condition (without uncertainty visualization vs. stop light colors vs. geometric contrast vs. both uncertainty visualizations) as the between-subjects factor and uncertainty value (uncertain vs. medium vs. certain) as the within-subject factor was used. The results showed that appearance of certain content, its uncertainty values, and their verbal scientific justifications were remembered better than uncertain ones. Furthermore, stop light colors enhanced the memory of uncertainty values compared to no uncertainty visualization and were better understood, discriminated, and transferred than geometric contrast. Geometric contrast decreased the memory of the appearance of uncertain architectural elements compared to no uncertainty visualization and was better than stop light colors regarding the memory of the appearance of certain architectural elements. The study integrates and extends existing theories by showing that certain contents are processed with higher priority than uncertain contents and that the multimedia effect is also valid for metainformation such as the uncertainties of contents. Finally, recommendations for designing learning material including uncertainty visualizations are given.
\end{abstract}

Keywords Visualization · Uncertainty · Archeological reconstructions · Lexical hedges $\cdot$ Memory

Manuela Glaser

m.glaser@iwm-tuebingen.de

Extended author information available on the last page of the article 
In the past years, there has been a growing awareness to teach students not only the basic laws, models, and findings of various scientific subjects, but also the nature of science (NOS), including its empirical and inferential approach, its grounding in theory, and its social embeddedness (Abd-El-Khalick et al., 2017; Osborne et al., 2003). Within this context, the tentativeness of scientific claims is an important topic, not only meaning that science is tentative in principle, because claims may be falsified in the future by new evidence, but also that particular scientific claims are usually made with varying degrees of uncertainty (Kampourakis \& McCain, 2020). In order to understand scientific information adequately, to engage in informed discussions, to form an opinion, and to make decisions based on the given information, the degree of uncertainty of the information and the nature of possible uncertainties must be presented, elaborated, and taken into account (Kirschenbaum \& Arruda, 1994; Leitner \& Buttenfield, 2000; Padilla, et al., 2020). But despite these claims, it has been shown that school textbooks and undergraduate textbooks tend to emphasize factuality and downplay tentativeness and uncertainty (Abd-El-Khalick et al., 2017; Radojčić, 2019). The effects of expressing uncertainty on source credibility and attitude formation have attracted some research in the field of science communication to the public, and the effects of uncertainty visualizations on preference, interpretability, and decision accuracy have been examined in fields such as geographic information science, meteorology, or medicine. However, corresponding research on these effects on learning and knowledge acquisition is scarce. Therefore, the present study asks how verbally and visually expressing different degrees of uncertainty of information influences learning.

\section{Types of Uncertainty in Science Communication and Education}

When we think of study material in textbooks or in online learning resources, we first think of the provision of clear, undisputed facts. But if we take a closer look, we find that in many fields, not all of the information that is conveyed is completely certain and that this uncertainty is often not explicitly communicated. By taking into account that information can be uncertain for a variety of reasons, several classification schemes have been proposed.

According to Gershon (1998), information may be uncertain because it can either be corrupt, incomplete, inconsistent, inaccurate, inferred or predicted with only a particular probability, or the source of the information can be of reduced credibility. Skeels et al. (2010) have grouped these different sources of uncertainty into three levels: at the lowest level, uncertainty results from imprecise measurements; at the middle level, uncertainty results from incompleteness due to sampling methods, missing values, or data aggregation; at the highest level, uncertainty results from inferences spanning all types of modelling, prediction of future events, and extrapolation into the past. Across all three levels, credibility of information and disagreement constitute two additional sources of uncertainty. Similarly, Gustafson and Rice (2020) distinguish between scientific uncertainty, consensus uncertainty, deficient uncertainty, and technical uncertainty. Scientific uncertainty addresses the tentative nature of scientific knowledge due to the possibility of new evidence in 
the future; consensus uncertainty addresses diverging or contradictory accounts for scientific phenomena; deficient uncertainty acknowledges evidential gaps in scientific research; and technical uncertainty addresses the fact that scientific claims are limited by measurement errors, modelling approximation, and statistical assumptions. In a similar vein, Spiegelhalter (2017) distinguishes aleatoric uncertainty (i.e., the unpredictability of future events which we cannot know and which is similar to the unknown unknowns and scientific uncertainty by Gustafson \& Rice, 2020) from epistemic uncertainty (uncertainty from a lack of knowledge that could theoretically be reduced given more information which we currently do not know but theoretically could and which is similar to deficient uncertainty by Gustafson \& Rice, 2020), and ontological uncertainty (uncertainty about how accurately the modelling describes reality which can only be described subjectively).

\section{Expressing Uncertainty in Learning Materials}

Taking the different types of uncertainty into account, van der Bles et al. (2019) have proposed a framework for communicating epistemic uncertainty to an audience. Based on Lasswell's (1948) general framework of media communication, they decompose the communication process into who communicates what kind of epistemic uncertainty in what form to whom and to what effect. In other words, a sender of epistemic uncertainty information (who) communicates about an issue for which there is uncertainty in terms of facts, numbers, or scientific models and hypotheses, about the sources of these epistemic uncertainties which are the reasons for the lack of knowledge, about the level of these epistemic uncertainties ranging from direct uncertainty about a fact to the indirect uncertainty, about a lack of confidence in the underlying science, and what magnitude these have (what). The sender communicates these epistemic uncertainties in a particular format (either numerical, verbal, or visual) and in a particular medium, such as scientific articles, scientific textbooks, popular science magazines or science documentaries on television (in what form). The recipients (to whom) constitute an audience with particular characteristics that are taken into account by the sender both in terms of communicated content and its form. As a result, communicating epistemic uncertainty leads to effects with regard to the cognition, emotion, trust, behavior, and decision making on the side of the recipient (to what effect).

As an example of applying this framework to educational contexts, the authors (who) of a school textbook (in what form) on geography may provide a chapter on weather forecasts together with a description of various sources of epistemic uncertainties such as measurement errors or different types of calculation methods and their implications for predicting the course of hurricanes (what). The epistemic uncertainty information is presented via written explanations including numerical probabilities in combination with graphs and charts containing visual markers of epistemic uncertainty (in what form). The text is used in 9th grade classes (to whom) and is intended to increase their knowledge and understanding of the complex interplay of environmental conditions, including the comprehension of the tentative status of forecasting models (to what effect). Another example would be a multimedia 
learning module on religious architecture in ancient Greece that includes temple reconstructions from archeological evidence together with verbal and visual expressions of the differing probabilities of various architectural elements, developed for a student audience with the goal of providing them an introduction to this topic, simultaneously making them aware of the epistemic uncertainties of architectural reconstructions.

Language provides a broad variety of verbal expressions called lexical hedges (Jensen, 2008). Among them are epistemic modality markers (Vold, 2006) for evaluating statements and therefore describe uncertainties in qualitative terms. Lexical hedges include modal verbs such as "could" or "would," lexical verbs like "guess" or "suppose," adverbs like "certainly" or "possibly," nouns such as "chance" or "opinion," and adjectives such as "sure" or "evident" (Crismore \& Vande Kopple, 1988; Holmes, 1988; Hyland, 1996). These seemingly fuzzy terms can be associated with particular degrees of uncertainty, at least at a group level (low, medium, high certainty), and can be empirically mapped onto a numerical scale ranging from 0 to 1 point or from 0 to $100 \%$ (Teigen \& Brun, 2003). However, the more distinct the probability levels one wishes to express are, the harder it is to do so (Erev \& Cohen, 1990).

Similarly, various graphical forms of expressing uncertainties have been developed, particularly in the field of geoscience (MacEachren et al., 2012). Typically, they are comprised of ordered sets of categories, visualized by abstract (e.g., those that are geometric, varying only a single visual variable such as location, size, or shape) or as iconic (e.g., those that are associative or pictorial, prompting metaphors such as stop light colors, bulls eye consistency, or smileys) representations of uncertainty. Also, both, verbal and visual indications of uncertainty are often combined in multimedia materials promising a better understanding of uncertainty (Glaser \& Schwan, 2019; Lengyel \& Toulouse, 2021).

While studies on lexical hedges in scientific texts show that lexical hedges are used rather frequently-every 50 words or in every 2 to 3 sentences (Hyland, 1996) — and Thiebach et al. (2015) have reported similar frequencies for popular scientific texts, it has been shown that school textbooks and undergraduate textbooks tend to emphasize factuality and to downplay tentativeness and uncertainty, as indicated by linguistic analyses (Abd-El-Khalick et al., 2017; Radojčić, 2019). It has therefore been argued that teaching should place more emphasis on issues of the nature of science and that the status of uncertainty of particular scientific claims, findings, and theories should be explicitly acknowledged in textbooks and other learning materials (Abd-El-Khalick et al., 2017; Osborne et al, 2003). This may be done by an adequate and thorough use of verbal and visual expressions of uncertainty.

\section{Processing Verbal Expressions of Uncertainty}

Most empirical studies on processing and the effects of verbally expressing uncertainty have been conducted in the field of public science communication, mainly addressing two aspects, namely, how indications of uncertainty and tentativeness 
influence recipients' perceived credibility of a message's source and content, and how they influence recipients' beliefs, opinions, and attitudes about the issues thematized in the message (Gustafson \& Rice, 2020; Kimmerle et al., 2015), while effects on knowledge acquisition and memory are relatively scarce. Research has typically focused on "hot topics" which are either disputed in public (like climate change) or have direct personal, most often health-related implications (like cancer, vaccination, or homeopathy).

Usually, readers of a popular science texts recognize these lexical hedges and are able to describe the uncertainty expressed in a text (Kimmerle et al., 2015). Therefore, lexical hedges may exert some influence on the subsequent processing of the information given. However, how uncertainty information is processed by recipients partly depends on its concrete formulation. For example, non-experts are often unaware of what threshold evidence is classified as high or low in uncertainty (van der Bles et al., 2019). Accordingly, combinations of verbal and numerical expressions of uncertainty may be better interpretable than words or numbers alone. In particular, the use of formulations such as "high uncertainty" alone has been found to lead to lower performance accuracy in decision making, lower satisfaction, and higher risk perception than the use of numbers, and therefore it is recommended to combine both forms of presentation (Zipkin et al., 2014). In turn, words as evaluative labels may provide an appropriate context to numerical uncertainty expressions (Spiegelhalter, 2017).

According to a recent review, expressions of technical uncertainty and, to a lesser degree, scientific uncertainty led to an increase in beliefs in, perceived credibility of, and intentions to follow the recommendations by a message (Gustafson \& Rice, 2020). In contrast, the reverse effects were found for expressions of consensus uncertainty, while the effects of expressions of deficient uncertainty were mixed in this regard. For example, Jensen (2008) found that authors of a scientific text were perceived as more trustworthy when lexical hedges indicating deficient uncertainty were used, whereas Thiebach et al. (2015) did not find effects of lexical hedges on the perceived scientificness of texts.

In the context of learning and knowledge acquisition, the effects of expressions of uncertainty on processing depth, elaboration, and memory are of particular importance. But, to the best of our knowledge, only few studies have addressed these issues to date. With regard to the influence of lexical hedges on the memory of scientific claims, Steffens et al. (2014) found only weak evidence. Mayweg-Paus and Jucks (2015), on the other hand, reported that, compared to texts without lexical hedges, texts expressing uncertainties of the presented information by lexical hedges were processed in more depth and that text statements were named more often in a subsequent essay task. These studies used text material that included different numbers of arguments and examined their effects only on the global level of the texts as a whole. Glaser and Schwan (2019), on the other hand, compared the influence of uncertainly versus certainly formulated arguments in an archeological scientific text with regard to perceived certainty and memory for the scientific contents on an argument level. Their results showed that textual scientific information presented with formulations of certainty were better retained than textual scientific information presented with formulations of uncertainty. The authors conclude that learners 
tend to give more weight to information marked as certain compared to information marked as uncertain, thus processing certain information more deeply according to their goal of establishing a coherent mental model of the scientific topic.

\section{Processing Visual Expressions of Uncertainty}

Besides verbal expressions, uncertainty may also be expressed visually. Similar to verbal expressions, there is also a rich repertoire of visual cues to signal uncertainty (MacEachren et al., 2012). Therefore, in many contexts-from scientific publications and mass media reports to learning materials-visualizations of uncertainty are used to make the confidence one has in a certain claim or piece of evidence more transparent to the recipients. But while verbal expressions are usually familiar from everyday contexts, the meaning of various visual cues of uncertainty used in scientific contexts may not be immediately apparent for laypeople. Accordingly, research has focused on different types of uncertainty visualizations with regard to instant identification, discrimination of different levels of uncertainty, and appropriate interpretation (Kosara et al., 2002; MacEachren et al., 2012; Sanyal et al., 2009; Schweizer \& Goodchild, 1992; Xie et al., 2006).

Examining both abstract and iconic representations of uncertainty in maps, MacEachren et. al. (2012) found that some types were more intuitively comprehensible than others. The authors assumed that abstract symbol sets should be fast to judge since the process of interpreting order and direction is largely a perceptual task, while iconic symbol sets require more cognitive processing to identify the intended metaphorical relationship with the uncertainty condition signified. However, in case of a successful design of a set of visual uncertainty markers, the rating of the intuitiveness should be higher for iconic than for abstract symbol sets. The results partly support these assumptions. Overall, iconic symbol sets were rated slightly higher on intuitiveness than abstract symbol sets, while with regard to assessment accuracy, abstract and iconic symbol sets were judged equally well.

Selection of an appropriate number of uncertainty levels is also of importance since discriminability of different degrees of uncertainty is impeded with the number of uncertainty degrees represented (Erev \& Cohen, 1990). Windhager et al. (2019) discuss the trade-off between the omission of uncertainty information at different levels leading to the deception of the recipients and visual complexity that overstrains the cognitive capacities of the recipients. In line with this, Correll et al. (2018) showed that when using color scales to represent values or uncertainty, performance accuracy in an identification task is better with a discrete than with a continuous scale, indicating a trade-off between quantization error with using discrete scales of inherently continuous variables and perception error with continuous scales.

There are also several perceptual and cognitive biases that go along with the processing of uncertainty visualizations. With regard to visualizations of a particular kind of epistemic uncertainties, namely, statistical uncertainties, Padilla et al. (2020) argue for the use of icon arrays (displays in which one shape is repeated a number of times and some of the shapes are altered to represent a proportion) instead 
of percentages because icon arrays provide frequency framing and are therefore better understandable than percentages with their probabilistic framing. They further assume that the deterministic construal error (the attempt to substitute visual uncertainty information for deterministic information) can be avoided by animations of uncertainty that give the recipient an intuitive sense of uncertainty in the true meaning. Furthermore, Padilla et al. (2020) argue for ensemble displays or other error bar alternatives to avoid having recipients believe that visual boundaries of the uncertainty visualization are cognitive categories (Padilla et al., 2018). Finally, they highlight the use of visual semiotics of uncertainty (MacEachren et al., 2012), such as fuzziness, transparency, or stop light colors, because these allow the recipients to build on previous experience (Blackwell, 2001) and are therefore intuitive to understand by natural mapping. They also refer to the use of visualization techniques that do not allow the recipients to precisely look at uncertain values, so-called valuesuppressing uncertainty palettes. In contrast to bivariate color maps in which data visualization and uncertainty visualization are independent from each other, valuesuppressing uncertainty palettes couple both types of information by visualizing data that are more uncertain with a lower resolution than data that are more certain. Correll et al. (2018) showed that value-suppressing uncertainty palettes compared to traditional bivariate maps are able to focus the recipients' attention to data of high certainty and reduce discrimination of data of low certainty, thereby promoting riskavoiding compared to risk-seeking decision making.

To sum up, in many contexts, visualizations of uncertainty are used to make the confidence one has in a certain claim or piece of evidence more transparent to the recipients. And although visual expressions of uncertainty may complement verbal expressions of uncertainty, care should be taken in choosing the optimal type of uncertainty visualization since visualization styles differ with regard to their discriminability and understandability and influence also data processing by data suppression and visual and cognitive biases.

\section{Processing Learning Material with Verbal and Visual Expressions of Uncertainty}

To the best of our knowledge, until now, no studies exist that went beyond questions of identification, discrimination, and interpretation and have investigated the impact of uncertainty visualizations - alone or in combination with verbal expressions of uncertainty_on memory, learning, and knowledge acquisition. Given that learning implies the building of a coherent mental representation of a given content, what kind of impact does uncertainty information have on this process?

The knowledge generation model for visual analytics including uncertainty propagation and human trust building proposed by Sacha et al. (2016) may serve as a starting point for conceptualizing knowledge acquisition of learning content that is marked as being uncertain. The model not only describes how uncertainties are technically propagated through different steps of a data visualizing system-from the data source to the model and to the data visualization-but also describes how knowledge is built based on the awareness of uncertainty markers and trust building 
cues. In particular, the authors identify three different loops of a recipient's cognitive processing, namely, an exploration loop, a verification loop, and a knowledge generation loop.

At first, recipients approach the content with a certain amount of initial trust together with some first content-related hypotheses based on their prior knowledge. In the exploration loop, the presented information is searched through for evidence for or against both initial trust and initial hypotheses. If uncertainties are explicitly stated and the recipients have become aware of them, erroneous interpretation should decrease and the users' trust in the data should increase. In the verification loop, the recipients' initial hypotheses are refined by the evidence gained in the exploration loop. For example, a weakly trusted hypothesis will originate inspection of the presentation in an exploratory fashion that solidifies the hypotheses step by step, whereas a highly trusted hypothesis will lead to a more confirmatory approach. Finally, in the knowledge generation loop, gained insights that exceed a particular trust value will be integrated in the recipients' mental model of the learning content and transferred into long-term memory. These insights can match or contradict the initial mental model of the learning content and therefore either confirm, complement, or change it. They may also lead to an awareness of knowledge gaps and stimulate new hypotheses.

Crucial for the exploration loop is that learners both become aware of uncertainty information in the learning material and hold it in working memory, because only under these conditions, uncertainty information may exert an influence on the learning process. The research reviewed above shows that readers usually recognize lexical hedges in texts (Kimmerle et al., 2015), while recognition and correct interpretation of visual markers of uncertainty strongly depend on their particular design influencing discriminability and understandability (MacEachren et al., 2012). In addition, although theories of multimedia learning (Mayer, 2014) do not explicitly consider processing of uncertainty information, several of its principles may be valid for these cases as well. In particular, the multimedia principle (Butcher, 2014) may hold true such that uncertainties presented in the text will be better processed when accompanied by uncertainty visualizations because of a dual channel processing advantage. In contrast, simultaneous use of two uncertainty visualization styles should be less beneficial compared to the use of a single uncertainty visualization because of redundancy and cluttering placing unnecessary demands on cognitive resources. Therefore, it can be hypothesized that learners tend to take notice of verbal expressions of uncertainty and that their effect on further information processing is most pronounced when combined with appropriately designed visual expressions of uncertainty.

In order to successfully proceed through the verification loop, both content and corresponding uncertainty information have to be held in working memory. Then the contents are evaluated, taking into account their uncertainty values. According to Gilbert (1991), mental systems represent information in working memory per default as being "true" and then actively assess it with regard to its "real" truth value in a second step. In addition, according to recent models of comprehension, incoming content information is routinely monitored for its epistemic status of plausibility and (in-)validated accordingly (Lombardi et al., 2016; Richter \& Maier, 2017). 
Importantly, these models assume that the perceived plausibility of claims serves as a heuristic for cognitive resource allocation, with implausible information receiving less strategic and resource-intensive comprehension processes (Richter \& Maier, 2017). In its most extreme case, information is discarded from further processing if it falls below a minimum level of plausibility. Plausibility, defined as "potential truthfulness" (Lombardi et al., 2016), bears a close similarity to the concept of uncertainty, meaning that given information may be true but only with a particular probability. Also, both plausibility and uncertainty span a wide range from highly implausible or uncertain to highly plausible or nearly certain. The main difference between the two fields of research lies in the explicitness of the cues of plausibility and uncertainty. While models of epistemic monitoring are concerned with inferring plausibility from various bits of information, research on uncertainty is concerned with the impact that verbal and visual expressions explicitly stating degrees of uncertainty have on information processing. Therefore, due to the commonalities of plausibility and uncertainty, comprehension models may provide a theoretical framework that can be tentatively applied to explicit statements of uncertainty as well. In consequence, it can be hypothesized that learners tend to process learning content marked as highly certain more deeply than learning content marked as less certain and to even discard processing learning content marked as being highly uncertain. In line with this assumption, Glaser and Schwan (2019) found that learners of archeological scientific articles learned scientific claims better if they were formulated as being certain compared to uncertain.

Finally, Sacha et al. (2016) assume that during the knowledge generation loop, the outcome of the verification loop that exceeds a particular trust value is integrated by the help of prior knowledge, connected to existing knowledge structures and permanently stored in long-term memory. Ideally, the resulting mental model not only includes a coherent representation of the various elements of the learning content but also includes additional information about their respective uncertainties. Psycholinguistic models of comprehension of negation suggest that the association of content elements and epistemic markers may follow a "schema plus tag" principle; that is, irrespective of its epistemic status, a proposition is stored as a factual statement, which is then qualified by a link to its epistemic status (e.g., its negation or its degree of uncertainty; Mayo et al., 2004). Because the memory linkage between the content elements and the uncertainty tags may get lost over time, uncertain content elements tend to be falsely remembered as being certain more often than vice versa (Pandelaere \& Dewitte, 2006). Similarly, in the context of communication research, Hovland and Weiss (1951) showed that participants discounted information from untrustworthy sources at the time of exposure, but over time dissociated the content and the source leading to a decline in the original skepticism and the acceptance of the untrustworthy information. Possibly, this memory bias may be prevented or delayed if the storage of uncertainty values in memory is fostered. If the multimedia effect (Butcher, 2014) can be generalized from learning content to uncertainty values, combinations of verbal and visual expressions of uncertainty should be better memorized and retained than verbal expressions of uncertainty alone (dual coding).

This integrated approach combining the knowledge generation model for visual analytics including uncertainty propagation and human trust building proposed by 
Sacha et al. (2016), the cognitive theory of multimedia learning by Mayer (2014), the multimedia principle (Butcher, 2014), and previous studies on the effects of different uncertainty visualization styles are visualized in Fig. 1.

In order to take a first step in validating this integrated model of processing evidence and its uncertainty values, the present study examines the influence of additional uncertainty visualizations on learning content and uncertainty values. Thereby, we focus on a particular field of science education in which uncertainty plays an outstanding role: archeology and ancient history. Here, too, different uncertainty visualization styles may influence the text-picture integration of the presented information and its uncertainties differently.

\section{The Present Study}

The present study used archeology and ancient history as a prototypical fields of education in which verbal and visual expressions of uncertainty play a prominent role. The main sources of evidence about ancient history are ancient texts and archeological excavations. Archeological evidence is often condensed into reconstructions that are not only used for information exchange, hypothesis formulation, and discussion among experts (Kinkeldey et al., 2015) but also in learning materials for students or laypersons, often accompanying textual descriptions (Kolenda \& Markiewicz, 2017). Usually, a reconstruction represents only one of several possible versions of an ancient place or building, and different parts of the reconstruction are of different certainties (Bernardes et al., 2012). Therefore, reconstructions include various degrees of what Gustafson and Rice (2020) call deficient uncertainty, and Spiegelhalter (2017) calls epistemic uncertainty. More specifically, different elements of a reconstruction may be based on different types of evidence ranging from direct evidence of excavated objects (low uncertainty) over indirect evidence by

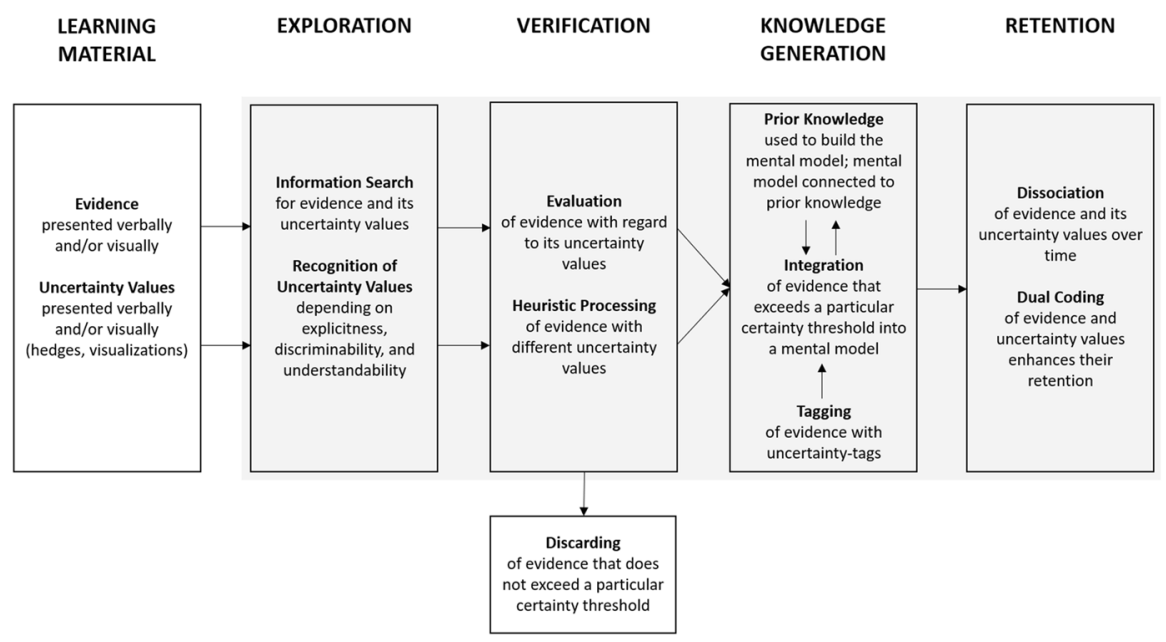

Fig. 1 Integrated model of processing evidence and its uncertainty values 
formal analogies with other ancient buildings (medium uncertainty) to weak evidence by functional analogies (high uncertainty; de Haan, 1999).

Accordingly, in order not to mislead the learners, the visualization of the reconstructions should indicate where archeological finds end and reconstruction begins (Sifniotis et al., 2007). Therefore, various techniques have been developed to indicate uncertainties in architectural reconstructions, including grayscale, geometric abstraction or geometric contrast (Lengyel \& Toulouse, 2011, 2015; van der Wel et al., 1994), thickness and style (dotted or solid) of lines (Boukhelifa et al., 2012; Gershon, 1998; Griethe \& Schumann, 2005; Strothotte et al, 1999), and also stop light colors (Apollonio, 2016; Apollonio \& Giovannini, 2015; Danielová et al., 2016). Apollonio (2016) posits that among the methods to visualize uncertainty in $3 \mathrm{D}$ reconstructions, the use of color is the most efficient method because it allows for understanding in a clear manner and according to widely shared semantic codes. Correspondingly, MacEachren et al. (2012) showed that attribute trustworthiness was best represented by stop light colors, leading to the highest assessment accuracy values compared to other visualizations. However, memory for the spatial layout of the three-dimensional scenery may be impaired by the overlay of color. As an alternative that accounts especially for the three-dimensionality of archeological reconstructions, geometric contrast (Lengyel \& Toulouse, 2015) shows architectural elements which are certain in a detailed manner, whereas uncertain elements are represented by simple geometric forms such as cuboids, balls, or cones. However, geometric contrast is a value-suppressing visualization style which does not visualize uncertain contents in detail and therefore may hinder learning or in combination with verbal explanations of the learning content may hinder dual coding of uncertain learning content. This should also be the case for combinations of geometric contrast with stop light colors. Hence, with geometric contrast compared to stop light colors, memory for the uncertain information should be worse while spatial memory of the scenery should be better.

By referring to the knowledge generation model for visual analytics including uncertainty propagation and human trust building (Sacha et al., 2016), we assume that learning ancient history via reconstructions depends on the perceived or elaborated uncertainty of the learning content. Learning content marked as being certain should be processed more deeply and therefore better consolidated in long-term memory than learning content marked as being uncertain. In addition, the processing of the uncertainty values associated with the learning contents should follow established principles of multimedia learning, leading to an advantage of combinations of verbal and visual uncertainty expressions compared to verbal uncertainty expressions alone (Butcher, 2014). But given the many different options of visualizing uncertainty, the type of uncertainty visualization may matter. Value-suppressing geometric contrast should be detrimental especially for learning uncertain learning contents. Stop light colors, on the other hand, should be easier to comprehend, discriminate, and transfer, thus being better for dual coding and therefore for the learning of uncertainty information compared to geometric contrast. Finally, the choice of uncertainty visualization style may also affect the memory for the spatial layout for the depicted scenery as well as the perception of esthetic and authenticity, which are examined exploratively. 
In order to test these assumptions, in the present study, the participants worked through a learning environment about the ancient city Pergamon that included two learning modules each about a particular religious temple in Pergamon along with a picture of the respective reconstruction and an accompanying audio explanation. In the audio explanations, the degree of uncertainty of the single reconstruction elements was expressed via lexical hedges. In the pictures of the reconstructions, the degree of uncertainty of the single reconstruction elements was either not expressed, expressed via stop light colors, via geometric contrast, or via a combination of both. Lexical hedges as well as the two visualization styles distinguished between three degrees of uncertainty, namely, low certainty, medium certainty, and high certainty. We measured memory for the learning content (appearance of architectural elements), memory for uncertainty values and their verbal scientific justifications, discriminability and understandability of the uncertainty visualizations, transfer of the understanding of the uncertainty visualization, and, exploratorily, spatial memory of the scenery, authenticity of the reconstruction, and esthetic of the presentation style and scenery as the dependent variables. We hypothesized that the appearance of architectural elements marked as being certain should be elaborated more deeply and therefore be better retained than the appearance of architectural elements marked as being uncertain (H1). We also hypothesized that low uncertainty values should be better retained than high uncertainty values (H2). Furthermore, uncertainty values should be better retained with than without uncertainty visualizations (H3). Comparing different types of uncertainty visualizations, we assumed that memory of the appearance of uncertain information should be worse with geometric contrast than with stop light colors (H4). We further assume stop light colors to be better discriminable, understandable, and transferable than geometric contrast (H5). With regard to these hypotheses, combining both types of visualization was expected to be less beneficial than each of them alone due to an increase of unnecessary redundancy and clutter (H6). Finally, we exploratorily asked whether stop light colors and geometric contrast influence spatial memory of the scenery differently (RQ1) and whether authenticity of the archeological reconstructions and esthetic of the presentation style and scenery are influenced by stop light colors and geometric contrast (RQ2). In Table 1, for each of these hypotheses and research questions, independent and dependent variables are summarized.

\section{Method}

\section{Participants}

Participants were recruited from our institute's mailing list which includes mostly students who are interested in taking part in our studies. They had to speak German on a native speaker level, must not be studying or have studied archeology, history, or architecture or be practicing similar professions, and must have no color ametropia in the conditions including stop light colors. The participants were from a broad variety of disciplines. Of the 147 recruited participants, 23 had to be excluded, either because they did not speak German on a native speaker level $(n=2)$, studied history 


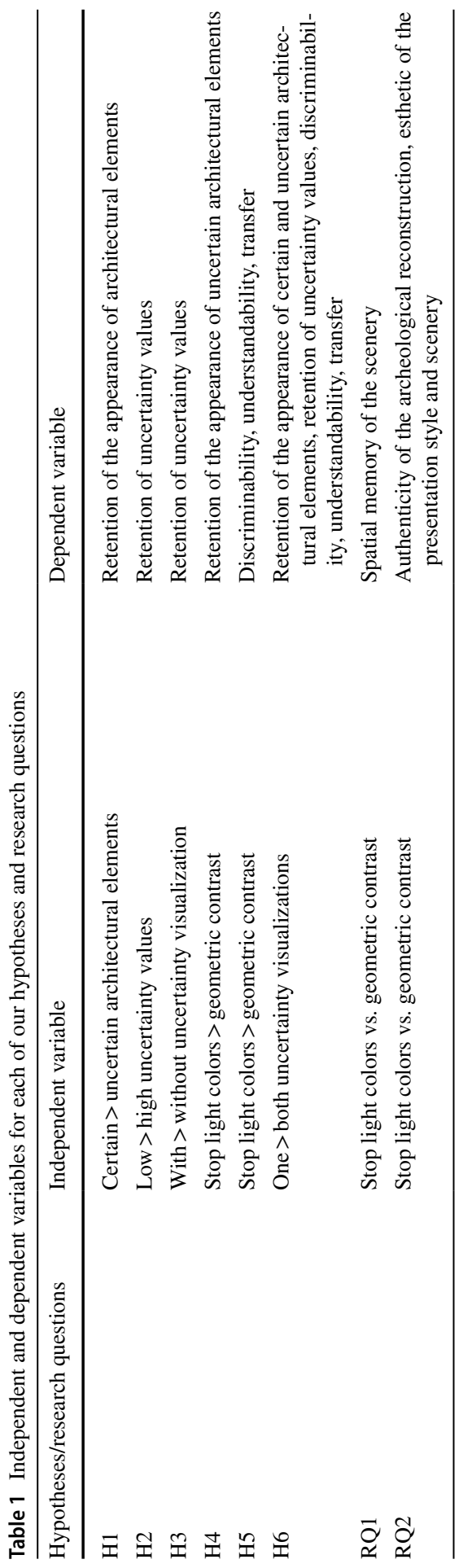


$(n=1)$, knew the research material from previous studies $(n=2)$, failed at least one of the manipulation checks $(n=10)$, had not understood the instructions $(n=2)$, or because of technical problems $(n=6)$. The remaining 124 participants were aged between 18 and 68 years $(M=26.41, \mathrm{SD}=9.70), 33(26.6 \%)$ of them were male, $90(72.6 \%)$ were female, and $1(0.8 \%)$ was undefined. Assuming medium effect sizes $(f=0.25)$, according to an a priori G-power analysis, exactly this sample size would have been needed for a power of $1-\beta=0.80$ with $\alpha=0.05$. The participants received ten Euros for their participation. They were randomly assigned to four conditions (without uncertainty visualization vs. stop light colors vs. geometric contrast vs. both uncertainty visualizations).

\section{Materials}

\section{Learning Environment}

The participants were instructed to work through a computer-based, linear learning environment about the ancient city Pergamon and two of its most important buildings - the temple of Athena and the temple of Demeter. The learning environment contained text information, photographs, and maps, as well as pictures of reconstructions of each of the two temples with accompanying audio explanations. The participants were not able to navigate backwards within the learning environment. Graphical elements could be enlarged, and the temple reconstructions plus accompanying audio explanations played only once by mouse click. Watching the pictures of the temple reconstructions with accompanying audio explanations was mandatory. They had to be played in full length and could not be paused or stopped.

\section{Pictures of Reconstructions with Accompanying Audio Explanations}

There were two pictures of temple reconstructions with accompanying audio explanations in the learning environment - one about the architecture of the Athena temple and the other about the architecture of the Demeter temple. In addition to the picture of each reconstruction, an audio explanation referring to the various individual architectural elements was provided. The audio explanation also provided information about the uncertainty values of each of the architectural elements and the scientific justification for them. Of the twelve architectural elements of each temple, four were described as being uncertain, four as being of medium certainty, and four as being certain. Uncertainty values were expressed by lexical hedges representing uncertain (e.g., "maybe"), medium (e.g., "highly probable"), and certain (e.g., "definitely") values and corresponding scientific justifications representing either functional analogies (e.g., "because it looked more representative"), formal analogies (e.g., "because on other Athena temples in the same region the architectural element looked alike"), or findings (e.g., "because remains of the architectural element were found").

In order to create the experimental conditions, the pictures of the reconstructions were manipulated, whereas the audio explanation was the same in all conditions. For 
the condition without any uncertainty visualization, each reconstruction was presented in grey scale and full detail. For the condition with only color coding, each reconstruction was presented in full detail and its single architectural elements were colored. Uncertain architectural elements were colored in red, elements of medium certainty values were colored in yellow, and certain elements were colored in green. For the condition with only geometric contrast, each reconstruction was presented in grey scale, and the detailedness of the single architectural elements varied with their uncertainty value. Uncertain architectural elements were presented with a coarsegrained geometry similar to building blocks, elements of medium certainty were presented with a medium-grained geometry, and certain elements were presented with fine-grained geometry, analogous to the fully detailed version in the condition without any uncertainty visualization. For the condition with both types of uncertainty visualization-color coding and geometric contrast-the aforementioned uncertainty visualizations were combined. The reconstructions in the different conditions are presented in Fig. 2.

\section{Design}

We used a mixed $4 \times 3$ design with condition (without uncertainty visualization vs. stop light colors vs. geometric contrast vs. both uncertainty visualizations) as the between-subjects factor and uncertainty value (uncertain, medium, certain) as the within-subject-factor. Analyses were calculated across the two temple reconstructions.

\section{Measures}

\section{Prior Knowledge About Pergamon}

Prior knowledge about Pergamon was measured in order to check whether the participants in the different conditions a priori differed with regard to this aspect. It was measured with one item ("How do you estimate your prior knowledge about Pergamon?") on a five-point Likert scale ranging from one ("no prior knowledge") to five ("very high prior knowledge").

\section{Manipulation Check}

In the three conditions with uncertainty visualization, understanding of the respective uncertainty visualization was measured by presenting a simple altar reconstruction in three uncertainty versions (uncertain, medium, certain) shown in the visualization style of the respective condition. The participants were asked to correctly indicate the visualized uncertainty value. Answers to all three uncertainty visualizations had to be correct to pass the manipulation check. 

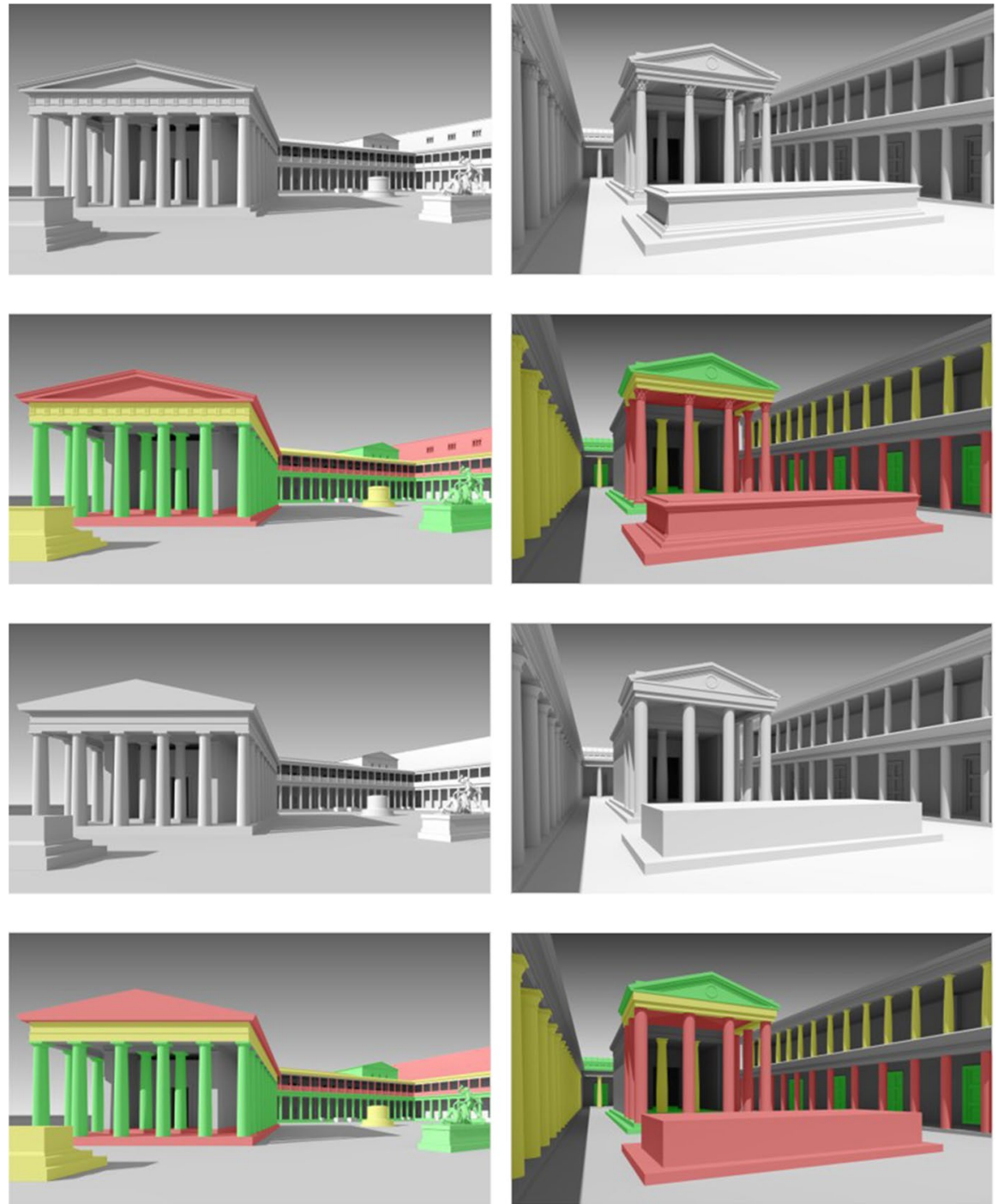

Fig. 2 Picture of the reconstruction of the architecture of the Athena Temple (left column) and the Demeter Temple (right column) in the condition without uncertainty visualization (first row), with stop light colors (second row), with geometric contrast (third row), and with both types of certainty visualization (fourth row)

\section{Memory of the Appearance of Architectural Elements}

The participants' memory of the appearance of the architectural elements of the temples was measured by a free recall test in paper-pencil form. They were asked to describe in note form the reconstruction of the Athena and the Demeter temple, including all its aspects and architectural elements that they could remember. 
Additionally, they were asked to complement this description by a sketch of the reconstruction, again including all aspects and architectural elements they could remember. There was no time limit for the participants. The free recall tests were analyzed by two independent raters on the basis of the twelve architectural elements mentioned in the audio explanation of the respective temple reconstruction, collapsing both description and sketch. Interrater reliability (Krippendorff's alpha) of the test scores was $\alpha=0.92$. In the case of inconsistent coding, the rating of a third rater was decisive. Each correctly recalled architectural element counted one point. Proportion correct scores for uncertain, medium, and certain architectural elements of each temple reconstruction were calculated.

\section{Memory of Uncertainty Values and Their Verbal Scientific Justifications}

For each of the twelve architectural elements of each of the temple reconstructions, the participants had to remember its uncertainty value by marking one of four categories (uncertain, medium, certain, don't know) and had to recall the verbal scientific justification of the particular uncertainty value in an open question. Answers on the scientific justifications were analyzed by two independent raters on the basis of the scientific justifications for the twelve architectural elements mentioned in the audio explanations of the respective temple reconstruction. Interrater reliability (Krippendorff's alpha) of the test scores was $\alpha=0.98$. In the case of inconsistent coding, the rating of a third rater was decisive. Each correctly recalled justification counted one point. A proportion correct score on remembered uncertainty values and a proportion correct score on remembered justification were calculated for uncertain, medium, and certain items of each temple reconstruction.

\section{Discriminability and Understandability of the Uncertainty Visualization}

In the three conditions with uncertainty visualization, discriminability and understandability of the uncertainty visualization were each measured by one item. Discriminability of the uncertainty visualization was measured with the question: "How well could you discriminate between these three levels?" on a five-point Likert scale ranging from one ("not at all") to five ("very well"). Understandability of the uncertainty visualization was measured with the question: "How subjectively understandable did you find this graphic rendition" also on a five-point Likert scale ranging from one ("not at all") to five ("very much").

\section{Transfer of the Understanding of the Uncertainty Visualization}

In the conditions with uncertainty visualization, transfer of the understanding of the uncertainty visualization was measured. The participants were shown a picture of a reconstruction of another temple in Pergamon-the Trajan temple-containing uncertainty visualizations corresponding to the respective condition. In addition to this picture, they were given three different written statements on the uncertainty of each of three architectural elements of the depicted temple reconstruction. The three statements differed with regard to the level of uncertainty (uncertain, medium, 
certain) that they ascribed to the particular architectural element by the use of hedges similar to the hedges in the audio explanations accompanying the pictures of the temple reconstructions. The participants had to indicate which of the written uncertainty statements corresponded to the uncertainty visualization in the picture (uncertain, medium, certain). Each correct answer was rated with one point. A proportion correct score across these three items was calculated for each participant.

\section{Spatial Memory of the Scenery}

In order to measure the participants' spatial memory of the scenery, they had to place and align a graphic representation of the camera angle used in the picture of the temple reconstruction on the ground views of the two temple areas. Placements were rated by two independent raters based on previously defined visual criteria. Interrater reliability (Krippendorff's alpha) was $\alpha=0.96$. Each correct placement was coded with one point. In case of inconsistent coding, a third rater made the decision.

\section{Authenticity of the Reconstructions}

Authenticity of each of the presented reconstructions was measured with a single item ("How authentic do you find the presented reconstruction of the temple?") on a five-point Likert scale ranging from one ("not at all") to five ("very much").

\section{Esthetic of Presentation Style and Scenery}

The esthetic of the presentation style with which the reconstructions of the two temples were presented was measured with a single item ("How esthetic do you find the presentation of the temple?") on a five-point Likert scale ranging from one ("not at all") to five ("very much"). In addition, the esthetic of each of the reconstructed architectures was also measured with a single item ("How esthetic do you find the presented scenery?") on a five-point Likert scale ranging from one ("not at all") to five ("very much"). These both esthetic values were analyzed separately.

\section{Procedure}

The participants were invited in groups of one to four participants. They were welcomed, and each participant was seated in front of a computer with a 27-inch display and a headset. They were instructed in written form on the computer screen to read the information in the learning environment thoroughly and to answer questions on it afterwards. They were requested to start the learning environment by mouse click. In the learning environment, the first slide presented text information about the city Pergamon, its location, its important buildings, and its history. This text was accompanied by related photographs, maps, and a timeline. The second slide provided text information about the temple of Athena, its location, and its area, as well as related photographs and maps. The third slide provided a text introduction about the 
uncertainty of the architecture of the Athena temple and a picture of its reconstruction with an accompanying audio explanation about its architecture, which the participants were instructed to start by mouse click. In the conditions with uncertainty visualizations, an additional text about the uncertainty visualization used in the picture of the temple reconstruction was presented, followed by the manipulation check of understanding the uncertainty visualization. Next, the temple reconstruction with the accompanying audio explanations had to be started by the participant. After the participants had watched the picture of the temple reconstruction with the accompanying audio explanations, slides about the temple of Demeter were presented, which were structured analogously to the slides about the Athena temple.

Finally, after finishing this learning phase, the participants had to complete the measures on discriminability and understandability of the uncertainty visualization (only in the conditions with uncertainty visualization). After that, the free recall test on the architecture of the temple (in paper pencil form), the retention test on the uncertainty values and verbal uncertainty justifications of the architectural elements of the temple, the spatial perception test with regard to the temple, the questions about the authenticity of the temple reconstruction, and the questions about the esthetic of the reconstruction and the scene of the sanctuary-first for the Athena and then for the Demeter temple. Then followed the transfer test, questions on demographic data and exclusion criteria (age, sex, field of study/profession, color ametropia, prior knowledge, knowledge of research material), and a debriefing about the fact that uncertainty values and their verbal scientific justifications were fictional due to experimental reasons. The study lasted approximately $75 \mathrm{~min}$ and received institutional research ethics committee approval.

\section{Data Analyses}

Analyses were calculated with SPSS version 25. Post-hoc tests of all the calculated ANOVAs were Bonferroni corrected to control for type 1 errors. The confidence intervals for the significant post-hoc tests are provided in Table 2 in the Appendix. The effect size $\eta^{2}$ for the Kruskal Wallis test on spatial memory of the scenery was calculated according to Lenhard and Lenhard (2016).

\section{Results}

\section{Prior Knowledge About Pergamon}

A one-factorial analysis of variance (ANOVA) with condition (without uncertainty visualization vs. stop light colors vs. geometric contrast vs. both uncertainty visualizations) as the between-subjects factor was calculated with regard to prior knowledge about Pergamon and revealed no significant differences between the conditions, $F(3,123)=0.79, p=0.504, \eta_{p}{ }^{2}=0.019$. The participants in the conditions without uncertainty visualization $(M=1.15, \mathrm{SD}=0.44)$, with stop light colors $(M=1.23, \mathrm{SD}=0.62)$, with geometric contrast $(M=1.19, \mathrm{SD}=0.48)$, and with both 
Table 2 Calculated analysis, their significant effects, their significant post-hoc comparisons, and corresponding confidence intervals

\begin{tabular}{|c|c|c|}
\hline Significant effects & $\begin{array}{l}\text { Significant post-hoc com- } \\
\text { parisons }\end{array}$ & $\begin{array}{l}\text { Confidence } \\
\text { interval } 95 \%\end{array}$ \\
\hline \multicolumn{3}{|c|}{ Mixed ANOVA: memory of the appearance of architectural elements } \\
\hline \multirow[t]{3}{*}{ Main effect of uncertainty value } & Certain $>$ uncertain & {$\left[\begin{array}{lll}0.230 & 0.315\end{array}\right]$} \\
\hline & Uncertain $>$ medium & {$[0.0350 .111]$} \\
\hline & Certain $>$ medium & {$\left[\begin{array}{lll}0.309 & 0.383\end{array}\right]$} \\
\hline Main effect of condition & Without $>$ both & {$\left[\begin{array}{lll}0.063 & 0.288\end{array}\right]$} \\
\hline \multirow[t]{23}{*}{ Interaction } & Without: & \\
\hline & Certain $>$ uncertain & {$\left[\begin{array}{lll}0.023 & 0.189\end{array}\right]$} \\
\hline & Uncertain $>$ medium & {$\left[\begin{array}{lll}0.089 & 0.237\end{array}\right]$} \\
\hline & Certain $>$ medium & {$\left[\begin{array}{lll}0.197 & 0.341]\end{array}\right.$} \\
\hline & Stop light colors: & \\
\hline & Certain $>$ uncertain & {$\left[\begin{array}{lll}0.064 & 0.235\end{array}\right]$} \\
\hline & Uncertain $>$ medium & {$\left[\begin{array}{lll}0.053 & 0.205\end{array}\right]$} \\
\hline & Certain $>$ medium & {$\left[\begin{array}{lll}0.204 & 0.352\end{array}\right]$} \\
\hline & Geometric contrast: & \\
\hline & Certain $>$ uncertain & {$\left[\begin{array}{lll}0.370 & 0.541]\end{array}\right.$} \\
\hline & Certain $>$ medium & {$\left[\begin{array}{lll}0.370 & 0.517\end{array}\right]$} \\
\hline & Both: & \\
\hline & Certain $>$ uncertain & {$\left[\begin{array}{lll}0.291 & 0.468\end{array}\right]$} \\
\hline & Certain $>$ medium & [0.316 0.469$]$ \\
\hline & Uncertain: & \\
\hline & $\begin{array}{l}\text { Without }>\text { geometric } \\
\text { contrast }\end{array}$ & {$\left[\begin{array}{lll}0.118 & 0.392\end{array}\right]$} \\
\hline & $\begin{array}{l}\text { Stop light colors }>\text { geometric } \\
\text { contrast }\end{array}$ & {$\left[\begin{array}{lll}0.014 & 0.292\end{array}\right]$} \\
\hline & Without $>$ both & {$[0.1770 .456]$} \\
\hline & Stop light colors $>$ both & {$\left[\begin{array}{lll}0.073 & 0.356\end{array}\right]$} \\
\hline & Medium: & \\
\hline & Without $>$ both & {$\left[\begin{array}{lll}0.058 & 0.275\end{array}\right]$} \\
\hline & Certain: & \\
\hline & $\begin{array}{l}\text { Geometric contrast }>\text { stop } \\
\text { light colors }\end{array}$ & {$\left[\begin{array}{lll}0.006 & 0.300\end{array}\right]$} \\
\hline \multicolumn{3}{|c|}{ Mixed ANOVA: memory of uncertainty values } \\
\hline \multirow[t]{3}{*}{ Main effect of uncertainty value } & Certain $>$ medium & {$\left[\begin{array}{lll}0.147 & 0.252\end{array}\right]$} \\
\hline & Medium $>$ uncertain & {$\left[\begin{array}{lll}0.137 & 0.244\end{array}\right]$} \\
\hline & Certain $>$ uncertain & {$\left[\begin{array}{lll}0.334 & 0.446\end{array}\right]$} \\
\hline \multirow[t]{2}{*}{ Main effect of condition } & Stop light colors $>$ without & {$\left[\begin{array}{lll}0.069 & 0.252\end{array}\right]$} \\
\hline & Both $>$ without & {$\left[\begin{array}{lll}0.043 & 0.229\end{array}\right]$} \\
\hline
\end{tabular}


Table 2 (Continued)

\begin{tabular}{|c|c|c|}
\hline Significant effects & $\begin{array}{l}\text { Significant post-hoc com- } \\
\text { parisons }\end{array}$ & $\begin{array}{l}\text { Confidence } \\
\text { interval } 95 \%\end{array}$ \\
\hline \multicolumn{3}{|c|}{ Mixed ANOVA: memory of verbal scientific justifications } \\
\hline \multirow[t]{3}{*}{ Main effect of uncertainty value } & Certain $>$ medium & {$\left[\begin{array}{lll}0.110 & 0.210\end{array}\right]$} \\
\hline & Medium $>$ uncertain & {$\left[\begin{array}{lll}0.138 & 0.242\end{array}\right]$} \\
\hline & Certain $>$ uncertain & {$\left[\begin{array}{lll}0.291 & 0.409\end{array}\right]$} \\
\hline \multicolumn{3}{|c|}{ One-factorial ANOVA: discriminability } \\
\hline \multirow[t]{2}{*}{ Main effect of condition } & $\begin{array}{l}\text { Stop light colors }>\text { geometric } \\
\text { contrast }\end{array}$ & {$[0.6351 .365]$} \\
\hline & Both $>$ geometric contrast & {$\left[\begin{array}{lll}0.618 & 1.360\end{array}\right]$} \\
\hline \multicolumn{3}{|c|}{ One-factorial ANOVA: understandability } \\
\hline \multirow[t]{2}{*}{ Main effect of condition } & $\begin{array}{l}\text { Stop light colors }>\text { geometric } \\
\text { contrast }\end{array}$ & {$[0.3021 .376]$} \\
\hline & Both $>$ geometric contrast & {$[0.1621 .255]$} \\
\hline \multicolumn{3}{|l|}{ One-factorial ANOVA: transfer } \\
\hline \multirow[t]{2}{*}{ Main effect of condition } & $\begin{array}{l}\text { Stop light colors }>\text { geometric } \\
\text { contrast }\end{array}$ & {$\left[\begin{array}{lll}0.138 & 0.378\end{array}\right]$} \\
\hline & Both $>$ geometric contrast & {$\left[\begin{array}{lll}0.186 & 0.430\end{array}\right]$} \\
\hline
\end{tabular}

uncertainty visualizations $(M=1.38, \mathrm{SD}=0.86)$ had an equally low amount of prior knowledge about Pergamon.

\section{Memory of the Appearance of Architectural Elements}

An analysis of variance (ANOVA) with condition (without uncertainty visualization vs. stop light colors vs. geometric contrast vs. both uncertainty visualizations) as the between-subjects factor and uncertainty value (uncertain, medium, certain) as the within-subject factor was calculated with regard to the retention of the appearance of the architectural elements as described in the audio explanation. The results showed a significant main effect of uncertainty value, $F(2,240)=252.625$, $p<0.001, \eta_{p}{ }^{2}=0.678$. Bonferroni corrected post-hoc tests showed that the appearance of certain architectural elements $(M=0.53, \mathrm{SD}=0.22)$ was significantly better retained than the appearance of uncertain architectural elements $(M=0.26$, $\mathrm{SD}=0.24, p<0.001)$, and these were again better retained than architectural elements of medium certainty $(M=0.18, \mathrm{SD}=0.17, p<0.001)$. Furthermore, there was a significant main effect of condition, $F(3,120)=5.876, p<0.001, \eta_{p}{ }^{2}=0.128$. Bonferroni corrected post-hoc tests showed that the retention of the appearance of the architectural elements was significantly better without uncertainty visualization $(M=0.41, \mathrm{SD}=0.17)$ than with both uncertainty visualizations $(M=0.23$, $\mathrm{SD}=0.17, p<0.001)$-with stop light colors $(M=0.33, \mathrm{SD}=0.17)$ and geometric contrast $(M=0.33, \mathrm{SD}=0.17)$ lying in between.

There was also a significant interaction between uncertainty value and condition, $F(6,240)=14.147, p<0.001, \eta_{p}{ }^{2}=0.261$ (see Fig. 3). Bonferroni-corrected 


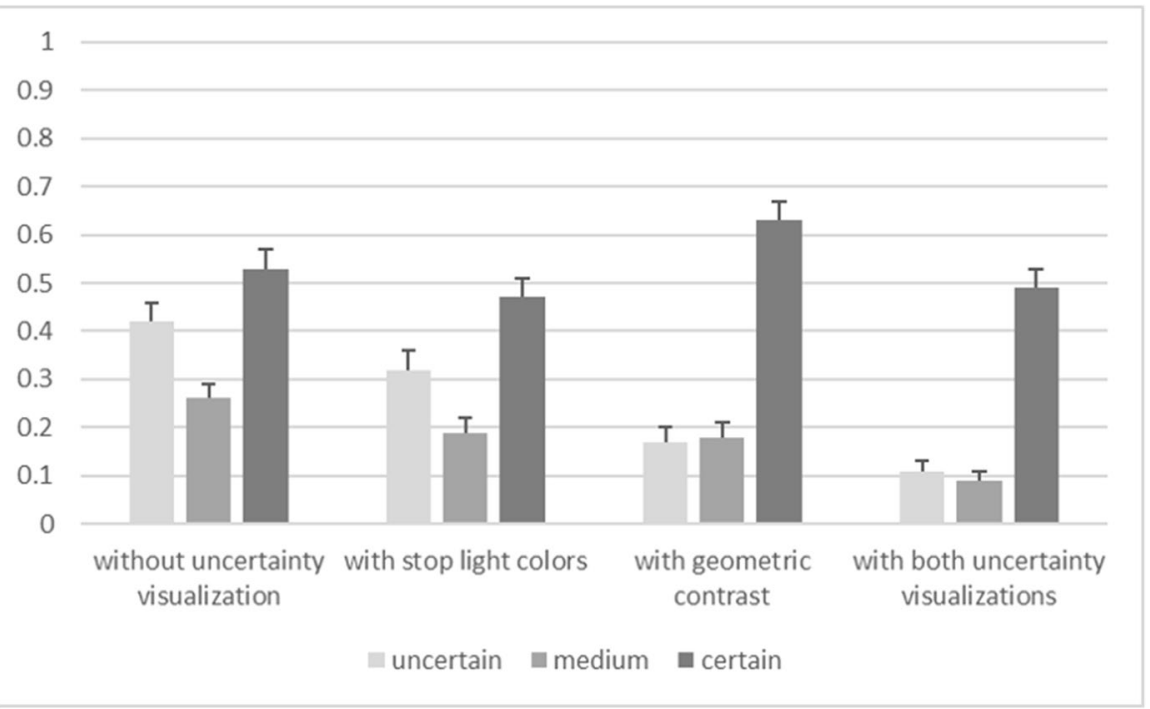

Fig. 3 Interaction between condition and uncertainty value with regard to memory of architectural appearance. Error bars indicate standard errors

post-hoc tests showed that the above-described main effect of the uncertainty value was present in the condition without any uncertainty visualization $(p=0.007$ between uncertain and certain architectural elements, all other $p<0.001)$ and in the condition with only stop light colors (all $p<0.001$ ). In the conditions with only geometric contrast and both uncertainty visualizations, this effect also occurred but without any significant difference between the uncertain architectural elements and the elements of medium certainty (both $p=1.000$ ). The appearance of uncertain architectural elements was significantly worse retained with geometric contrast $(M=0.17, \mathrm{SD}=0.17)$ compared to the condition without any uncertainty visualization $(M=0.42, \mathrm{SD}=0.25, p<0.001)$ and compared to the condition with only stop light colors $(M=0.32, \mathrm{SD}=0.23, p=0.023)$. Retention of details of uncertain architectural elements in the condition with both uncertainty visualizations $(M=0.11$, $\mathrm{SD}=0.13$ ) was also significantly worse than in the condition without any uncertainty visualization $(p<0.001)$ and worse than in the condition with only stop light colors $(p<0.001)$. Memory of the appearance of certain architectural elements was significantly higher with geometric contrast $(M=0.63, \mathrm{SD}=0.21)$ than with stop light colors $(M=0.47, \mathrm{SD}=0.23, p=0.036)$-with the conditions without uncertainty visualization $(M=0.53, \mathrm{SD}=0.23)$ and with both uncertainty visualizations $(M=0.49, \mathrm{SD}=0.19)$ lying in between. Memory of the appearance of architectural elements of medium certainty was significantly higher in the condition without uncertainty visualization $(M=0.26, \mathrm{SD}=0.20)$ than in the condition with both uncertainty visualizations $(M=0.09, \mathrm{SD}=0.10, p<0.001)$-with geometric contrast $(M=0.18, \mathrm{SD}=0.14)$ and stop light colors $(M=0.19, \mathrm{SD}=0.17)$ lying in between. There were no other significant post-hoc tests in this analysis. 


\section{Memory of Uncertainty Values and Their Verbal Scientific Justifications}

Analyses of variance (ANOVA) with condition (without uncertainty visualization vs. stop light colors vs. geometric contrast vs. both uncertainty visualizations) as the between-subjects factor and uncertainty value (uncertain vs. medium vs. certain) as the within-subject factor were calculated with regard to the retention of uncertainty values and the retention of the verbal uncertainty justification.

With regard to the retention of the uncertainty values, the results revealed a significant main effect of the uncertainty value, $F(2,240)=153.467, p<0.001$, $\eta_{p}{ }^{2}=0.561$. Bonferroni-corrected post-hoc tests showed that uncertainty values of certain architectural elements $(M=0.61, \mathrm{SD}=0.21)$ were significantly better retained than uncertainty values of architectural elements of medium certainty $(M=0.41$, $\mathrm{SD}=0.21$ ), and these were again significantly better retained than the uncertainty values of uncertain architectural elements $(M=0.22, \mathrm{SD}=0.19) \quad(p$ 's $<0.001)$. There was also a significant main effect of condition, $F(3,120)=8.615, p<0.001$,

$\eta_{p}{ }^{2}=0.177$. Bonferroni-corrected post-hoc tests showed that stop light colors alone $(M=0.48, \mathrm{SD}=0.14)$ as well as both uncertainty visualizations together $(M=0.46$, $\mathrm{SD}=0.13$ ) each significantly improved the memory of uncertainty values compared to the condition without uncertainty visualization $(M=0.32, \mathrm{SD}=0.14)$ ( $p$ 's $<0.001)$, while geometric contrast $(M=0.41, \mathrm{SD}=0.14)$ did not differ significantly from the condition without uncertainty visualization. There were no other significant post-hoc tests in this analysis, and there was also no significant interaction between both factors.

With regard to the retention of the verbal scientific justification of the uncertainty values of the architectural elements, the results showed a significant main effect of the uncertainty value, $F(2,240)=125.549, p<0.001, \eta_{p}{ }^{2}=0.511$. Bonferroni-corrected post-hoc tests showed that verbal scientific justifications of the uncertainty values of certain architectural elements $(M=0.47, \mathrm{SD}=0.28)$ were significantly better retained than those of the uncertainty values of architectural elements of medium certainty $(M=0.31, \mathrm{SD}=0.23)(p<0.001)$, and these were again significantly better retained than the those of the uncertainty values of uncertain architectural elements $(M=0.12, \mathrm{SD}=0.16)(p<0.001)$. There were no other significant main effects or interactions in this analysis.

\section{Discriminability and Understandability of the Uncertainty Visualization}

One-factorial analyses of variance (ANOVA) with condition (stop light colors vs. geometric contrast vs. both uncertainty visualizations) as the between-subjects factor were calculated with regard to discriminability and understandability of the uncertainty visualization. The results revealed a significant effect of condition on discriminability, $F(2,90)=29.15, p<0.001, \eta_{p}{ }^{2}=0.398$. Bonferroni-corrected posthoc tests showed that the three levels of the uncertainty visualization could be discriminated better with stop light colors alone $(M=4.84, \mathrm{SD}=0.45)$ as well as with both uncertainty visualizations $(M=4.83, \mathrm{SD}=0.38)$ each compared to geometric 
contrast $(M=3.84, \mathrm{SD}=0.82)(p$ 's $<0.001)$. There was also a significant effect of condition on understandability, $F(2,90)=8.37, p<0.001, \eta_{p}{ }^{2}=0.160$. Again, Bonferroni-corrected post-hoc tests showed that stop light colors $(M=4.61, \mathrm{SD}=0.67$, $p<0.001)$ and both uncertainty visualizations $(M=4.48, \mathrm{SD}=0.91, p=0.006)$ were each judged to be better understandable than geometric contrast alone $(M=3.77$, $\mathrm{SD}=0.99)$.

\section{Transfer of the Understanding of the Uncertainty Visualization}

A one-factorial analyses of variance (ANOVA) with condition (stop light colors vs. geometric contrast vs. both uncertainty visualizations) as the between-subjects factor was calculated with regard to the transfer of the understanding of the uncertainty visualization to another reconstruction example. The results revealed a significant effect of condition on transfer, $F(2,90)=22.27, p<0.001, \eta_{p}{ }^{2}=0.336$. Bonferronicorrected post-hoc tests showed that the transfer of the understanding of the uncertainty visualization was better for conditions with stop light colors alone $(M=0.89$, $\mathrm{SD}=0.20)$, as well as with both uncertainty visualizations $(M=0.94, \mathrm{SD}=0.16)$, each compared to the condition with geometric contrast $(M=0.63, \mathrm{SD}=0.22)$ $(p$ 's $<0.001)$.

\section{Spatial Memory of the Scenery}

Due to the dichotomous character of the answer scale of the spatial memory test, a Kruskal-Wallis test with condition (without uncertainty visualization vs. stop light colors vs. geometric contrast vs. both uncertainty visualizations) as the between-subjects factor was calculated with regard to spatial memory of the scenery. The results showed no significant main effect of condition, $H(3)=5.096$, $p=0.165, \eta^{2}=0.02$. Spatial memory was similar without uncertainty visualization $(M=0.45, \mathrm{SD}=0.42)$, with stop light colors $(M=0.47, \mathrm{SD}=0.41)$, with geometric contrast $(M=0.65, \mathrm{SD}=0.45)$, and with both uncertainty visualizations $(M=0.41, \mathrm{SD}=0.45)$.

\section{Authenticity of the Reconstructions}

A one-factorial analyses of variance (ANOVA) with condition (without uncertainty visualization vs. stop light colors vs. geometric contrast vs. both uncertainty visualizations) as the between-subjects factor were calculated with regard to the authenticity of the reconstructions. The results showed no significant main effect of condition, $F(3,120)=0.522, p=0.668, \eta_{p}{ }^{2}=0.013$. The reconstructions were rated as being equally authentic without uncertainty visualization $(M=3.99$, $\mathrm{SD}=0.93)$, with stop light colors $(M=3.74, \mathrm{SD}=0.80)$, with geometric contrast $(M=3.84, \mathrm{SD}=0.69)$, and with both uncertainty visualizations $(M=3.85$, $\mathrm{SD}=0.78)$. 


\section{Esthetic of the Presentation Style and Scenery}

One-factorial analyses of variance (ANOVA) with condition (without uncertainty visualization vs. stop light colors vs. geometric contrast vs. both uncertainty visualizations) as the between-subjects factor were calculated with regard to the esthetic of the presentation style and with regard to the esthetic of the scenery. The results with regard to the esthetic of the presentation style showed no significant main effect of condition, $F(3,120)=0.385, p=0.764, \eta_{p}{ }^{2}=0.010$. The presentation style was rated as being equally esthetic without uncertainty visualization $(M=3.56, \mathrm{SD}=1.07)$, with stop light colors $(M=3.58, \mathrm{SD}=0.92)$, with geometric contrast $(M=3.34, \mathrm{SD}=1.06)$, and with both uncertainty visualizations $(M=3.50, \mathrm{SD}=0.88)$. The results with regard to the esthetic of the scenery also showed no significant main effect of condition, $F(3,120)=0.322, p=0.810$, $\eta_{p}{ }^{2}=0.008$. The scenery was rated as being equally esthetic without uncertainty visualization $(M=3.48, \mathrm{SD}=1.09)$, with stop light colors $(M=3.29, \mathrm{SD}=0.98)$, with geometric contrast $(M=3.42, \mathrm{SD}=0.96)$, and with both uncertainty visualizations $(M=3.28, \mathrm{SD}=0.95)$.

\section{Discussion}

In the present paper, the role of verbal and visual signaling of uncertainty of learning contents was examined. By summing up recent literature on this topic, an integrated model of processing evidence and its uncertainty values, including three different processing steps (exploration, verification, knowledge generation) was developed. In addition, the influence of different uncertainty visualizations supplementing the verbal markers of uncertainty was studied. This was done by analyzing the influence of different degrees of information uncertainty (uncertain, medium, certain) and different uncertainty visualization styles (without uncertainty visualization, stop light colors, geometric contrast, both uncertainty visualizations) on processing pictures of archeological reconstructions with accompanying audio explanations presented in a learning environment about the ancient city Pergamon.

The results showed that the appearance of content marked as certain (H1), its uncertainty values (H2), and their verbal scientific justifications were remembered better than content marked as uncertain. Furthermore, stop light colors, but not geometric contrast alone, enhanced the memory of uncertainty values compared to no uncertainty visualization (H3). Geometric contrast decreased the memory of the appearance of uncertain architectural elements compared to no uncertainty visualization (H4) and was better than stop light colors regarding the memory of the appearance of certain architectural elements. Stop light colors were better understood, discriminated, and transferred than geometric contrast (H5). The assumption that combining both types of visualizations would be less beneficial than using each of the visualizations alone due to unnecessary redundancy and clutter (H6) could not be confirmed. Our exploratory research questions on whether the two uncertainty 
visualizations influence spatial memory of the scenery differently (RQ1) and on whether authenticity of the archeological reconstruction and esthetic of the presentation style and scenery are influenced by stop light colors and geometric contrast (RQ2) also showed no effects.

\section{Processing Uncertainty Information}

The results showed that independent of the presence and type of uncertainty visualizations, the appearance of architectural elements marked as certain was significantly better retained than the appearance of architectural elements marked as being of medium certainty or uncertain, confirming hypothesis H1. The percentage of remembered details dropped from over $50 \%$ for certain elements to about $25 \%$ for the uncertain elements. In addition, the retention of uncertainty values, as well as their verbal scientific justifications, was also better for architectural elements marked as certain than for elements marked as being of medium certainty or uncertain, confirming hypothesis H2. Again, this was a strong effect, with percentage correct dropping from $61 \%$ for values of certainty to $41 \%$ from values of medium certainty to $22 \%$ for values of uncertainty. Similarly, memory of verbal scientific justifications of uncertainties dropped from $47 \%$ for values of certainty to $31 \%$ for values of medium certainty to $12 \%$ for values of uncertainty. Taken together, this pattern of results indicates that the learners recognized expressions of uncertainty and took this information into account during learning. More specifically, learners seem to have used verbal and visual markers of uncertainty as heuristic cues for either selecting information for further processing or discarding it from processing instead. This holds true both for the appearance of architectural elements, for uncertainty values, and for verbal scientific justifications of these uncertainty values; that is, if a particular architectural element was marked as being uncertain, the architectural appearance, the uncertainty value, and its verbal scientific justification had a low probability of being processed further and transferred into long-term memory. In contrast, if a particular architectural element was marked as being certain, the architectural appearance, the uncertainty value, and its verbal scientific justification had a high probability of being processed further and transferred into long-term memory.

\section{Supplementing Verbal Naming of Uncertainties with Uncertainty Visualizations}

Regarding the influence of uncertainty visualizations on the memory of the appearance of the architectural elements, as well as the uncertainty values and their verbal scientific justification of these elements, the following results were found: Supplementing the verbal naming of uncertainties with additional uncertainty visualizations affected the retention of the appearance of the architectural elements dependent on the uncertainty level of these elements. In the case of uncertain architectural elements, the memory of their appearance was worse with geometric contrast or combinations of geometric contrast and stop light colors, each compared to stop 
light colors and no uncertainty visualization, confirming H4. In contrast, in the case of certain architectural elements, the memory of their appearance was better with geometric contrast compared to stop light colors. With regard to the memory of the appearance of architectural elements of medium certainty, using a combination of both uncertainty visualizations was worse than presenting verbal uncertainties without any uncertainty visualization. These results argue also predominantly against H6.

Supplementing verbal naming of uncertainties with additional uncertainty visualizations further increased the memory of the uncertainty values of architectural elements, depending on the type of visualization used. More specifically, adding either stop light colors or combinations of stop light colors and geometric contrast increased the memory of uncertainty values of architectural elements, whereas geometric contrast did not improve memory compared to verbal naming of uncertainties alone. These results partially confirmed $\mathrm{H} 3$ and argue against $\mathrm{H} 6$.

These differences between the uncertainty visualizations were also reflected in the subjective ratings of discriminability and understandability of the levels of uncertainty, confirming H5 and again argue against H6. Furthermore, stop light colors and combinations of stop light colors with geometric contrast were rated as being better discriminable, better understandable, and led to a better transfer to new instances of architectural elements.

Taken together, the pattern of results indicates that stop light colors saliently signal uncertainty values, whereas learners have difficulty noticing and identifying uncertainty values provided by geometric contrast as a prototype of value-suppressing visualizations (Correll et al., 2018). In line with Boukhelifa et al. (2012), the three uncertainty levels represented by stop light colors could easily be discriminated and understood by the learners. The findings also confirm the assumptions and results of previous research by Apollonio (2016) as well as MacEachren et al. (2012) emphasizing the potential of stop light colors for interpretability. The present study additionally showed - to the best of our knowledge for the first time - that the ability to interpret and understand stop light colors as representations of uncertainty values was also transferred to new learning content.

Furthermore, stop light colors and geometric contrast had opposing effects on the memory of the appearance of architectural elements and their uncertainty values. Stop light colors increased the memory of uncertainty values but did not affect the memory of the appearance of architectural elements. In contrast, in the case of geometric contrast, the memory of uncertainty values was not affected, but the memory of the appearance of architectural elements was dependent on the degree of uncertainty of the archeological elements. Memory of the appearance of uncertain archeological elements was decreased with geometric contrast compared to a verbal presentation of uncertainties without any uncertainty visualization due to its value-suppressing character (Correll et al., 2018). Memory of the appearance of certain architectural elements, on the other hand, was not enhanced by geometric contrast compared to verbal presentations without any uncertainty visualization but was only higher compared to the use of stop light colors. This confirms the original intention of the developers of geometric contrast (Lengyel \& Toulouse, 2015) to account for the three-dimensionality of the archeological reconstructions, compared 
to other visualization styles such as stop light colors, which may affect the visibility of details by covering them too.

The fact that, compared to stop light colors, geometric contrast was worse discriminated, understood, and transferred and also failed to positively affect the memory of uncertainty values may be due to the low strictness of its category borders. While the colors green, yellow, and red of the stop light color visualization are clearly perceivable and distinguishable, the borders between detailed, medium, and abstract visualization with geometric contrast are more fluent, especially with regard to architectures as the learning content. Learners may not always be sure whether a building block represents an abstraction of an archeological element or a detailed architectural element that has just a simple cuboid form such as modern cuboid seatings in front of official buildings or in pedestrian areas. Furthermore, while stop light colors and geometric contrast both are iconic forms of uncertainty visualizations, learners may be less familiar with geometric contrast than with stop light colors that are present nearly every day when they use their cars, bicycles, or participate in traffic as pedestrians. Future studies should therefore examine whether trainings such as repeated interpretation of information with geometric contrast as uncertainty visualization (e.g., through establishment of this type of uncertainty visualization in informal learning settings such as museums and exhibitions) or repeated decisionmaking tasks based on information presented with geometric contrast as uncertainty information (e.g., in formal learning settings such as the education of archeologists) can be used to enhance the efficiency of geometric contrast regarding discriminability, understandability, and transfer.

\section{Theoretical Implications}

According to the proposed integrated model of processing evidence and its uncertainty values, learners process uncertain information in three different steps: exploration, verification, and knowledge generation. During the exploration of the learning content, learners should become aware of uncertainty information and transfer it into working memory, because only under these conditions may uncertainty information exert an influence on the learning process. The present study demonstrates that learners indeed took notice of uncertainty information when it was presented by appropriately designed visualizations in a salient manner (MacEachren et al., 2012). Thus, combinations of verbal uncertainty expression with stop light color visualization or with a combination of stop light colors and geometric contrast outperformed verbal uncertainty expressions alone. In addition, this pattern of results indicates that the multimedia effect (Butcher, 2014) not only holds for the learning content itself but also for metainformation such as uncertainty information: uncertainty values were better learned when presented as text and picture compared to text alone. Hence, multimedia research should examine their design principles also with different types of metainformation that are important to be processed with regard to particular learning goals. Finally, while Kimmerle et al. (2015) found that learners recognize uncertainties regarding "hot topics" in public science communication that 
potentially have a high self-relevance (like health issues), the present findings show this also for neutral scientific topics presented in multimedia learning environments.

In the verification step, both content and corresponding uncertainty information should be held in working memory and related to each other. Recent models of comprehension assume that the perceived plausibility of claims serves as a heuristic for cognitive resource allocation (Lombardi et al., 2016; Richter \& Maier, 2017). Accordingly, the present study indicates that uncertain information received less strategic and resource-intensive comprehension processes, leading to worse retention of the appearance of architectural elements marked as being uncertain, their uncertainty values, and the verbal scientific justifications of these uncertainty values. Therefore, learners seem to have used uncertainty information as a heuristic cue for relevance or, as the case may be, irrelevance, discarding content marked as being uncertain from further processing (for a similar finding see Glaser \& Schwan, 2019). From an educational perspective, this constitutes a dilemma: On the one hand, for reasons of fostering understanding the nature of science, learners should be made aware of the varying uncertainties contained in a scientific account, like a report on archeological evidence studied in the present case. On the other hand, for reasons of content-related learning, learners should preferably process and elaborate all given information, irrespective of its status of uncertainty. We speculate that the learning goal may play an important role in this regard. For example, instead of having the goal to establish a coherent evidence-based mental model of a scientific topic, a different processing goal which focuses on uncertainties (for example, the goal of researchers to identify elements that need further scientific investigation) might lead to different processing strategies and outcomes. Similarly, with regard to decision making, uncertainty information or risk information may play a crucial role, and therefore uncertain information may be focused and processed with high priority.

Finally, in the knowledge generation step, learning content that exceeds a particular certainty threshold will be integrated in the recipient's mental model of the learning content and transferred into long-term memory. Ideally, the resulting mental model not only includes a coherent representation of the various elements of the learning content but also includes additional information about their respective uncertainties, at least for those contents that have passed the uncertainty threshold in the verification loop. Previous research indicates that uncertainty tags are added to processed information (Gilbert, 1991) and that these tags may get lost over time (Hovland \& Weiss, 1951; Mayo et al., 2004). From the perspective of multimedia learning and dual coding approaches, double presentation of learning content and uncertainty information via combinations of verbal expressions and visualizations should foster memory of the links between content and uncertainty values and therefore reduce dissociation of both types of information over time. The present study was not designed for addressing this issue, but future research should include delayed memory tests to examine the long-term memory for learning content and uncertainty tags, depending on the different types of marking uncertainties verbally and visually.

There are also some limitations of the present study. First, with regard to our explorative research questions, our results did not show any effect of uncertainty visualization on the spatial memory of the scenery, authenticity of the reconstructions, 
or esthetic of the presentation style and scenery. This may be due to methodological reasons. We used a measure which assessed the spatial memory of the scenery on a dichotomous scale without representing the memory of the scenery on a finergrained level, which may have allowed us to detect possible effects better. Future studies should therefore use more appropriate measures with answer scales on an interval level to examine the influence of uncertainty visualizations on the spatial memory of the scenery. Additionally, spatial memory of the scenery, authenticity of the reconstructions, as well as esthetic of the presentation style and scenery were all measured with one item. We may not have assessed these complex concepts with sufficient differentiation. Future studies should therefore use whole scales to examine the influence of uncertainty visualizations on these concepts. Finally, our results are only valid for learners with low prior knowledge. Future studies should therefore examine potential differences between laypersons and experts learning or working with archeological reconstructions or other learning material in which uncertainty information is provided verbally and/or visually.

Practitioners may pick up the results of the present study and integrate them in their processes of designing materials for science communication to the public as well as to students in formal learning contexts. This includes especially the visualization of uncertainty information to make dual coding processes possible and thereby enhance the learning of uncertainty information. Furthermore, it also includes the choice of an appropriate visualization style. Here, differences of uncertainty visualizations with regard to discriminability, understandability, and transferability should be taken into account. Finally, it should be decided carefully whether to use value suppressing or a non-valuesuppressing visualization styles. This decision should depend on the particular learning goal as well as the appropriate degree of uncertainty levels. But while the present study has identified the problem of using uncertainty as a heuristic cue for irrelevance, further empirical research is needed to determine how to solve this issue, for example, by appropriate instructional design, for example, an instruction which formulates and thereby enhances awareness of the bias to neglect uncertain information.

To conclude, the present study integrates research from different fields, such as communication research, psychology, and geographical information science, and confirms as well as extends theoretical assumptions about communicating and processing scientific information for learning scenarios (Butcher, 2014; Mayer, 2014; Mayo et al., 2014; Sacha et al., 2016). It integrates the models and empirical findings into a model comprised of an exploration step, a verification step, and a knowledge generation step. Finally, it demonstrates that the metainformation of learning contents such as uncertainty information, as well as its visualization, has a significant impact on the exploration and verification step, with corresponding consequences for learning outcomes. The present study thereby highlights the importance of considering uncertainty information and other aspects of the nature of science in communicating and processing science information and provides recommendations for practitioners in the field of science learning. 
Funding Open Access funding enabled and organized by Projekt DEAL.

\section{Declarations}

Ethics Approval All procedures performed in the study involving human participation were in accordance with the ethical standards of the DGPs (Deutsche Gesellschaft für Psychologie, German Psychological Society) and the APA (American Psychological Association) and have been approved by the local Institutional Review Board.

Consent to Participate Informed consent was obtained from all individual participants included in the study.

Conflict of Interest The authors declare no competing interests.

Open Access This article is licensed under a Creative Commons Attribution 4.0 International License, which permits use, sharing, adaptation, distribution and reproduction in any medium or format, as long as you give appropriate credit to the original author(s) and the source, provide a link to the Creative Commons licence, and indicate if changes were made. The images or other third party material in this article are included in the article's Creative Commons licence, unless indicated otherwise in a credit line to the material. If material is not included in the article's Creative Commons licence and your intended use is not permitted by statutory regulation or exceeds the permitted use, you will need to obtain permission directly from the copyright holder. To view a copy of this licence, visit http://creativecommons.org/ licenses/by/4.0/.

\section{References}

Abd-El-Khalick, F., Myers, J. Y., Summers, R., Brunner, J., Waight, N., Wahbeh, N., Zeinedding, A. A., \& Belarmino, J. (2017). A longitudinal analysis of the extent and manner of representations of nature of science in US high school biology and physics textbooks. Journal of Research in Science Teaching, 54(1), 82-120. https://doi.org/10.1002/tea.21339

Apollonio, F. I. (2016). Classification schemes for visualization of uncertainty in digital hypothetical reconstruction. In S. Münster, M. Pfarr-Harfst, P. Kuroczyński, \& M. Ioannides (eds.), 3D research challenges in cultural heritage II (pp. 173-197). Springer. https://doi.org/10.1007/ 978-3-319-47647-6_9

Apollonio, F. I., \& Giovannini, E. C. (2015). A paradata documentation methodology for the uncertainty visualization in digital reconstruction of $\mathrm{CH}$ artifacts. SCIRES-IT-SCIentific RESearch and Information Technology, 5(1), 1-24. https://doi.org/10.2423/i22394303v5n1p1

Bernardes, P., Madeira, J., Martins, M., \& Meireles, J. (2012). The use of traditional and computer-based visualization in archeology: A user survey. The 13th International Symposium on Virtual Reality, Archaeology and Cultural Heritage VAST, https://doi.org/10.2312/PE/VAST/VAST12S/013-016

Blackwell, A. F. (2001). Pictorial representation and metaphor in visual language design. Journal of Visual Languages \& Computing, 12(3), 223-252. https://doi.org/10.1006/jvlc.2001.0207

Boukhelifa, N., Bezerianos, A., \& Isenberg, T. (2012). Evaluating sketchiness as a visual variable for the depiction of qualitative uncertainty. IEEE Transactions on Visualization and Computer Graphics, 18, 2769-2778. https://doi.org/10.1109/TVCG.2012.220

Butcher, K. R. (2014). The multimedia principle. In R. E. Mayer (ed.), The Cambridge handbook of multimedia learning (pp. 174-205). Cambridge University Press. https://doi.org/10.1017/CBO9781139 547369.010

Correll, M., Moritz, D., \& Heer, J. (2018). Value-suppressing uncertainty palettes. Proceedings of the 2018 CHI Conference on Human Factors in Computing Systems (pp. 1-11). https://doi.org/10.1145/ 3173574.3174216

Crismore, A., \& Vande Kopple, W. J. (1988). Readers' learning from prose: The effects of hedges. Written Communication, 5(2), 184-202. https://doi.org/10.1177/0741088388005002004 
Danielová, M., Kumke, H., \& Peters, S. (2016). 3D reconstruction and uncertainty modelling using fuzzy logic of archaeological structures: Applied to the Temple of Diana in Nemi, Italy. Cartographica: The International Journal for Geographic Information and Geovisualization, 51(3), 137-146. https://doi.org/10.3138/cart.51.3.3160

De Haan, F. (1999). Evidentiality and epistemic modality: Setting boundaries. Southwest Journal of Linguistics, 18(1), 83-101.

Erev, I., \& Cohen, B. L. (1990). Verbal versus numerical probabilities: Efficiency, biases, and the preference paradox. Organizational Behavior and Human Decision Processes, 45, 1-18. https://doi.org/ 10.1016/0749-5978(90)90002-Q

Gershon, N. (1998). Visualization of an imperfect world. IEEE Computer Graphics and Application, 18, 43-45. https://doi.org/10.1109/38.689662

Gilbert, D. T. (1991). How Mental Systems Believe. American Psychologist, 46(2), 107-119. https://doi. org/10.1037/0003-066X.46.2.107

Glaser, M., \& Schwan, S. (2019). Processing textual and visual certainty information about digital architectural models. Computers in Human Behavior, 96, 141-148. https://doi.org/10.1016/j.chb.2019. 02.023

Griethe, H. \& Schumann, H. (2005). Visualizing uncertainty for improved decision making. Proceedings of the 4th International Conference on Perspectives in Business Informatics Research, University of Skovde, Sweden.

Gustafson, A., \& Rice, R. E. (2020). A review of the effects of uncertainty in public science communication. Public Understanding of Science, 29(6), 614-633. https://doi.org/10.1177/0963662520942122

Holmes, J. (1988). Doubt and certainty in ESL textbooks. Applied Linguistics, 9(1), 21-44. https://doi. org/10.1093/applin/9.1.21

Hovland, C. I., \& Weiss, W. (1951). The influence of source credibility on communication effectiveness. Public Opinion Quarterly, 15(4), 635-650. https://doi.org/10.1086/266350

Hyland, K. (1996). Talking to the academy: Forms of hedging in science research articles. Written Communication, 13(2), 251-281. https://doi.org/10.1177/0741088396013002004

Jensen, J. D. (2008). Scientific uncertainty in news coverage of cancer research: Effects of hedging on scientists' and journalists' credibility. Human Communication Research, 34(3), 347-369. https://doi. org/10.1111/j.1468-2958.2008.00324.x

Kampourakis, K., \& McCain, K. (2020). Uncertainty. Oxford University Press. https://doi.org/10.1093/ oso/9780190871666.001.0001

Kimmerle, J., Flemming, D., Feinkohl, I., \& Cress, U. (2015). How laypeople understand the tentativeness of medical research news in the media: An experimental study on the perception of information about deep brain stimulation. Science Communication, 37(2), 173-189. https://doi.org/10.1177/ 1075547014556541

Kinkeldey, C., Schiewe, J., Gerstmann, H., Götze, C., Kit, O., Lüdeke, M., Taubenböck, H., \& Wurm, M. (2015). Evaluating the use of uncertainty visualization for exploratory analysis of land cover change: A qualitative expert user study. Computers \& Geosciences, 84, 46-53. https://doi.org/10. 1016/j.cageo.2015.08.007

Kirschenbaum, S. S., \& Arruda, J. E. (1994). Effects of graphic and verbal probability information on command decision making. Human Factors, 36, 406-418. https://doi.org/10.1177/0018720894 03600302

Kolenda, J., \& Markiewicz, M. (2017). A medieval bishop's palace in Milicz: 3D reconstruction as a method of a research hypotheses presentation. Studies in Digital Heritage, 1(2), 428-443. https:// doi.org/10.14434/sdh.v1i2.23458

Kosara, R., Miksch, S., Hauser, H., Schrammel, J., Giller, V., \& Tscheligi, M. (2002). Useful properties of semantic depth of field for better $\mathrm{F}+\mathrm{C}$ visualization. In Proceedings of the symposium on Data Visualisation 2002 (pp. 205-210).

Lasswell, H. D. (1948). The structure and function of communication in society. In L. Bryson (Ed.), The communication of ideas (pp. 37-51). Harper.

Leitner, M., \& Buttenfield, B. P. (2000). Guidelines for the display of attribute certainty. Cartography and Geographic Information Science, 27, 3-14. https://doi.org/10.1559/152304000783548037

Lengyel, D. \& Toulouse, C. (2011). Darstellung von unscharfem Wissen in der Rekonstruktion historischer Bauten. In K. Heine, K. Rheidt, F. Henze, A. Riedel (eds.), Von Handaufmaß bis High Tech III. 3D in der historischen Bauforschung (pp. 182-186). Darmstadt/Mainz. 
Lengyel, D., \& Toulouse, C. (2015). The consecution of uncertain knowledge, hypotheses and the design of abstraction. Proceedings of the 20th International Conference on Cultural Heritage and New Technologies (pp. 1-16).

Lengyel, D. \& Toulouse, C. (2021). Experiencing architectonic design processes through interactive virtual reality applications. In V. Cappellini (ed.), Electronic imaging \& the visual arts: EVA 2021 (pp. 110-117). Florence. http://digital.casalini.it/9788859621591

Lenhard, W. \& Lenhard, A. (2016). Computation of effect sizes. Retrieved from: https://www.psychometr ica.de/effect_size.html. Psychometrica. DOI: https://doi.org/10.13140/RG.2.2.17823.92329

Lombardi, D., Nussbaum, E. M., \& Sinatra, G. M. (2016). Plausibility judgments in conceptual change and epistemic cognition. Educational Psychologist, 51(1), 35-56. https://doi.org/10.1080/00461520. 2015.1113134

MacEachren, A. M., Roth, R. E., O’Brien, J., Li, B., Swingley, D., \& Gahegan, M. (2012). Visual semiotics \& uncertainty visualization: An empirical study. IEEE Transactions on Visualization and Computer Graphics, 18(12), 2496-2505. https://doi.org/10.1109/TVCG.2012.279

Mayer, R. E. (2014). The Cambridge handbook of multimedia learning (2nd ed.). Cambridge University Press. https://doi.org/10.1017/CBO9781139547369

Mayo, R., Schul, Y., \& Burnstein, E. (2004). "I am not guilty" vs "I am innocent": Successful negation may depend on the schema used for its encoding. Journal of Experimental Social Psychology, 40(4), 433-449. https://doi.org/10.1016/j.jesp.2003.07.008

Mayo, R., Schul, Y., \& Rosenthal, M. (2014). If you negate, you may forget: Negated repetitions impair memory compared with affirmative repetitions. Journal of Experimental Psychology: General, 143(4), 1541-1552. https://doi.org/10.1037/a0036122

Mayweg-Paus, E., \& Jucks, R. (2015). Evident or doubtful? How lexical hints in written information influence laypersons' understanding of influenza. Psychology, Health \& Medicine, 20(8), 989-996. https://doi.org/10.1080/13548506.2014.986139

Osborne, J., Collins, S., Ratcliffe, M., Millar, R., \& Duschl, R. (2003). What "ideas-about-science" should be taught in school science? A Delphi study of the expert community. Journal of Research in Science Teaching, 40(7), 692-720. https://doi.org/10.1002/tea.10105

Padilla, L. M., Creem-Regehr, S. H., Hegarty, M., \& Stefanucci, J. K. (2018). Decision making with visualizations: A cognitive framework across disciplines. Cognitive Research: Principles and Implications, 3(1), 1-25. https://doi.org/10.1186/s41235-018-0120-9

Padilla, L., Kay, M., \& Hullman, J. (2020). Uncertainty visualization. https://doi.org/10.31234/osf.io/ ebd6r

Pandelaere, M., \& Dewitte, S. (2006). Is this a question? Not for long. The statement bias. Journal of Experimental Social Psychology, 42(4), 525-531. https://doi.org/10.1016/j.jesp.2005.08.004

Radojčić, S. (2019). Hedges and boosters in university textbooks. In P. Vičič, N. Gajšt, \& A. Plos (eds.), Conference Proceedings of the10th International Language Conference on "the Importance of Learning Professional Foreign Languages for Communication Between Cultures”, 20 and 21 September 2018, Celje, Slovenia (pp. 317-326). https://doi.org/10.18690/978-961-286-252-7.24

Richter, T., \& Maier, J. (2017). Comprehension of multiple documents with conflicting information: A two-step model of validation. Educational Psychologist, 52(3), 148-166. https://doi.org/10.1080/ 00461520.2017.1322968

Sacha, D., Senaratne, H., Kwon, B. C., Ellis, G., \& Keim, D. A. (2016). The role of uncertainty, awareness, and trust in visual analytics. IEEE Transactions on Visualization and Computer Graphics, 22(1), 240-249. https://doi.org/10.1109/TVCG.2015.2467591

Sanyal, J., Zhang, S., Bhattacharya, G., Amburn, P., \& Moorhead, R. J. (2009). IEEE Transactions on Visualization and Computer Graphics, 15, 1209-1218. https://doi.org/10.1109/TVCG.2009.114

Schweizer, D. M. \& Goodchild, M. F. (1992). Data quality and choropleth maps: An experiment with the use of color. Proceedings GIS/LIS 1992 (pp. 686-699). San Jose, California.

Sifniotis, M., Watten, P., Mania, K., \& White, M. (2007). Influencing factors on the visualisation of archaeological uncertainty. Proceedings of the 8th International conference on Virtual Reality, Archaeology and Intelligent Cultural Heritage (pp. 79-85).

Skeels, M., Lee, B., Smith, G., \& Robertson, G. G. (2010). Revealing uncertainty for information visualization. Information Visualization, 9, 70-81. https://doi.org/10.1057/ivs.2009.1

Spiegelhalter, D. (2017). Risk and uncertainty communication. Annual Review of Statistics and Its Application, 4, 31-60. https://doi.org/10.1146/annurev-statistics-010814-020148 
Steffens, B., Britt, M. A., Braasch, J. L., Strømsø, H., \& Bråten, I. (2014). Memory for scientific arguments and their sources: Claim-evidence consistency matters. Discourse Processes, 51(1-2), 117142. https://doi.org/10.1080/0163853X.2013.855868

Strothotte, T., Masuch, M., \& Isenberg, T. (1999). Visualizing knowledge about virtual reconstructions of ancient architecture. Proceedings Computer Graphics International (pp. 36-43). Los Alamitos, CA, The Computer Graphics Society, IEEE Computer Society. https://doi.org/10.1109/CGI.1999.777901

Teigen, K. H., \& Brun, W. (2003). Verbal probabilities: A question of frame? Journal of Behavioral Decision Making, 16(1), 53-72. https://doi.org/10.1002/bdm.432

Thiebach, M., Mayweg-Paus, E., \& Jucks, R. (2015). "Probably true" says the expert: How two types of lexical hedges influence students' evaluation of scientificness. European Journal of Psychology of Education, 30(3), 369-384. https://doi.org/10.1007/s10212-014-0243-4

van der Bles, A. M., van der Linden, S., Freeman, A. L., Mitchell, J., Galvao, A. B., Zaval, L., \& Spiegelhalter, D. J. (2019). Communicating uncertainty about facts, numbers and science. Royal Society Open Science, 6(5), 181870. https://doi.org/10.1098/rsos.181870

van der Wel, F. J. M., Hootsmans, R. M., \& Ormeling, F. (1994). Visualization of data quality. In: A. M. MacEachren \& D. R. Fraser Taylor (eds.), Visualization in modern cartography (pp. 313-331). Oxford: Pergamon. https://doi.org/10.1016/B978-0-08-042415-6.50023-5

Vold, E. T. (2006). Epistemic modality markers in research articles: A cross-linguistic and cross-disciplinary study. International Journal of Applied Linguistics, 16(1), 61-87. https://doi.org/10.1111/j. 1473-4192.2006.00106.x

Windhager, F., Salisu, S., \& Mayr, E. (2019). Exhibiting uncertainty: Visualizing data quality indicators for cultural collections. Informatics, 6(3), 29. https://doi.org/10.3390/informatics6030029

Xie, Z., Huang, S., Ward, M. O., \& Rundensteiner, E. A. (2006). Exploratory visualization of multivariate data with variable quality. IEEE Symposium on Visual Analytics Science and Technology (pp. 183 - 190). Baltimore. https://doi.org/10.1109/VAST.2006.261424

Zipkin, D. A., Umscheid, C. A., Keating, N. L., Allen, E., Aung, K., Beyth, R., Kaatz, S., Mann, D. M., Sussman, J. B., Korenstein, D., Schardt, C., Nagi, A., Sloane, R., \& Feldstein, D. A. (2014). Evidence-based risk communication: A systematic review. Annals of Internal Medicine, 161(4), 270-280. https://doi.org/10.7326/M14-0295

Publisher's Note Springer Nature remains neutral with regard to jurisdictional claims in published maps and institutional affiliations. 


\section{Authors and Affiliations}

\section{Manuela Glaser $^{1}$ (1) $\cdot$ Dominik Lengyel $^{2} \cdot$ Catherine Toulouse $^{2} \cdot$ Stephan Schwan $^{1}$}

Dominik Lengyel

lengyel@b-tu.de

Catherine Toulouse

toulouse@b-tu.de

Stephan Schwan

s.schwan@iwm-tuebingen.de

1 Leibniz-Institut für Wissensmedien, Tübingen, Germany

2 Brandenburgische Technische Universität Cottbus-Senftenberg, Cottbus, Germany 